\title{
Before the Jury Is out on Cinacalcet's Cardiovascular Effects in Hemodialysis Patients: Is Troponin a Missing Link?
}

\author{
Samra Abouchacra', Ahmed Chaaban', Mohammad Budruddin', Fares Chedid ${ }^{2}$, \\ Mohamad Hakim'1, Mohamad Ahmed', Nicole Gebran³ ${ }^{3}$ Farida Marzouki' \\ Muhy Eddin Hassan', Faiz Al Abbacheyi ${ }^{1}$ \\ ${ }^{1}$ Department of Medicine, Tawam Hospital, Abu Dhabi, UAE \\ ${ }^{2}$ Department of Pediatrics, Tawam Hospital, Abu Dhabi, UAE \\ ${ }^{3}$ Department of Pharmacology, Tawam Hospital, Abu Dhabi, UAE \\ ${ }^{4}$ SEHA Dialysis Services, Tawam Hospital, Abu Dhabi, UAE \\ Email: drbudruddin@gmail.com
}

Received 23 December 2013; revised 22 February 2014; accepted 10 March 2014

Copyright (C 2014 by authors and Scientific Research Publishing Inc.

This work is licensed under the Creative Commons Attribution International License (CC BY).

http://creativecommons.org/licenses/by/4.0/

(c) $\underset{\mathrm{EY}}{\mathrm{i}}$ Open Access

\section{Abstract}

Raised levels of the cardiac biomarker, Troponin I, are frequently encountered in hemodialysis patients and appear to be prognostic indicators for cardiovascular risk. Though evidence suggests that control of secondary hyperparathyroidism may reduce cardiac endpoints, the effect of the calcimimetic agent, cinacalcet, remains controversial. This retrospective study aimed at evaluating troponin levels in hemodialysis patients with severe secondary hyper parathyroidism (SHPT) who are on cinacalcet vs controls on conventional treatment. In addition, clinical outcomes including all-cause, cardiovascular morbidity and mortality were compared among both groups. A decline in Troponin I levels was observed in the cinacalcet group, this however was not translated clinically into improved survival. In fact, all-cause and cardiac mortality was similar in the two groups. Conversely, comparison of the incidence of cardiovascular events revealed lower rates in the cinacalcet group including cardiac, cerebral and peripheral vascular complications. Given some of our study limitations, further long-term, placebo-controlled trials are necessary to definitively establish the effect of cinacalet on cardiac biomarkers and ultimately its impact on clinical outcomes.

\section{Keywords}

Calcimimetics; Secondary Hyperparathyroidism; Parathyroid Hormone; Cinacalcet; Chronic Kidney Disease; Cardio Vascular Disease; End Stage Renal Disease; Troponin I; C-Reactive Protein; Parathyroidectomy; Acute Coronary Syndrome; 


\section{Introduction}

Increased cardiovascular morbidity and mortality are observed in chronic kidney disease (CKD) patients that have been attributed to traditional and uremia-related risks. The latter includes secondary hyperparathyroidism (SHPT) associated factors with multiple potential mechanisms, most notably related to vascular calcification [1] induced by abnormalities in calcium phosphate balance. This may directly lead to myocardial ischemia, finally culminating in overt cardiac disease, as secondary hyperparathyroidism progresses. Moreover, clinical observations support the etiologic role for parathyroid hormone (PTH) in the development of cardio vascular disease, whereby lowering its levels by parathyroidectomy, for instance, is associated with reduced vascular event rates and mortality in dialysis patients [2]. It has therefore been postulated that alternate measures to control SHPT and calcium-phosphate imbalance may impact myocardial ischemia and cardiac outcomes [3]. Most promising in its treatment is the emergence of the calcimimetic agent cinacalcet, which in addition to improving the biochemical parameters, has been anticipated to potentially impact cardiovascular outcomes [3].

On the other hand, since early detection is key to preemptive cardiovascular intervention, it follows that cardiac biomarkers' use is of tremendous clinical value, especially in CKD patients on dialysis. Though several cardiac peptides are available, troponin I appears more specifically for myocardial ischemia [4]-[7] and even serves as a prognostic indicators in dialysis patients [8]. It would hence be plausible that following troponin I trends may serve as possible markers of cinacalcet treatment effect on cardiac risk in these patients.

The aim of our study was to assess the effect of cinacalcet treatment on cardiac biomarker troponin I levels in patients on hemodialysis with severe SHPT. We also investigated the associated clinical outcomes in these patients compared to matched historical controls. The outcome measures included, in addition to overall and CV mortality, the composite CV event rates of myocardial infarction (MI), acute coronary syndrome (ACS), congestive heart failure (CHF), arrhythmia as well as cerebrovascular events (CVA) and peripheral vascular disease (PVD) complications.

\section{Study Design \& Methods}

This was a retrospective review of 246 hemodialysis patients in our center. The study was conducted between March 2012 until September 2013. Included were 71 adults with end stage renal disease on regular dialysis for $>6$ months with severe SHPT who had been on cinacalcet for at least 6 months. The control group was a historical matched cohort also with secondary hyperparathyroidism but of moderate severity who were maintained on vitamin D analogs and not receiving cinacalcet. Severe secondary hyperparathyroidism was defined as levels of PTH more than six times the upper limit of normal. Excluded were unstable patients and those in pediatric age group.

Baseline PTH, calcium, phosphate, C-Reactive Protien and Troponin I levels were retrieved and tracked over the two year period of the study. In our unit, PTH and troponin levels are routinely measured every three months. For the cinacalcet group, these parameters were obtained at baseline prior to starting the treatment with cinacalcet. Morbidity and mortality outcomes were followed for 6 months beyond the follow up period and included composite rates (MI, ACS, CHF, arrhythmia), CVA and PVD complications as well as sepsis-related admissions and overall mortality.

\section{Statistical Analysis}

Quantitative data were presented as mean (SD) or median (P25-P75) as appropriate, categorical data were presented as proportions. The basic characteristics, the laboratory results and the outcome variables were compared between the Cinacalcet group and the historical control with the two independent samples $t$ test or Mann Whitney $U$ test for measured data and Chi square tests or Fisher Exact test for nominal data. Changes in laboratory values in the Cinacalcet group overtime from baseline to 12 and 18 months were analyzed with repeated measure ANOVA when the assumptions of the test were met or the Friedman analysis of variance by ranks when the assumptions were not met (Troponin I levels). Further comparison PTH, Tropinin I, calcium and phosphorus between baseline and 18 months in patients of the Cinacalcet group who suffered a cardiac event was performed 
using with paired t test and its non-parametric alternative the Wilcoxin Sign Rank test as appropriate. All reported $P$ values were 2 -sided and deemed significant when they reached a $5 \%$ level. Statistical analysis was conducted using the PASW 19.0 statistical package (IBM SPSS, Chicago, Illinois).

\section{Results}

Out of 71 adult hemodialysis patients with severe secondary hyperparathyroidism who were on cinacalcet, we retrospectively reviewed 63 adult patients (30 Male; 33 Female) with complete data. Their mean age was $55.4 \pm$ 16 years with duration on HD ranging between 12 and 243 months averaging $74 \pm 58$ months. Associated co-morbidities, shown in (Table 1), included hypertension 78\%, diabetes 44\%, ischemic heart disease (IHD) $33 \%$. The control cohort was comprised of 40 adult patients (23 Male: 17 Female) with end stage renal disease on regular hemodialysis with moderately severe secondary hyperparathyroidism who were on Vitamin D or analogs but not receiving cinacalcet treatment. Their mean age was $60.4 \pm 15$ years and mean duration on HD of $46 \pm 89$ months with the following comorbid conditions: hypertension 80\%, diabetes 53\%, IHD 35\% (Table 1). None of the differences in baseline demographics achieved statistical significance except for dialysis vintage which was significantly longer in patients on cinacalcet $(\mathrm{p}<0.01)$.

PTH Levels decreased from baseline at 12 and 18 months, but the difference only achieved statistical significance with the latter interval. Similarly, although Troponin I levels tended to decrease at 12 months, a statistically significant decrement was noted only at 18 months. Conversely, calcium x phosphate product decreased significantly at both time periods however, C-reactive protein values decreased by $23 \%$ and $53 \%$ at 12 and 18 months respectively, but the difference did not reach statistical significance (Table 2).

Further statistical analysis was undertaken, where patients were paired serving as their own controls during follow up over the designated time periods. Data as shown in (Table 3) revealed a decrease in PTH levels at 12 and 18 months, the difference from baseline significant only at 18 months $(\mathrm{p}=0.026)$. In parallel, a statistically significant decrease of Troponin I was noted at 12 months (p 0.045), this however was not sustained at 18 months. CRP values decreased by $30 \%$ and $73 \%$, at 12 and 18 months respectively but the difference was not significant. One way repeated measure ANOVA comparing Calcium x Phosphate product at baseline, 12 and 18 months revealed a significant drop at both time points ( $\mathrm{p}<0.001)$.

Comparison of clinical outcome measures between patients receiving cinacalcet and the control group, revealed similar incidence of all-cause mortality of $10 \%$ in each. Cardiac mortality accounted for $50 \%$ in both groups, with no between group statistical difference. A comparison of the incidence of composite cardiac events revealed 33\% in the cinacalcet group versus 68\% in the control group as shown in (Table 4). This increased event rate in the controls was also observed for CVA and PVD complications although the latter approached yet did not achieve statistical significance $(p=0.07)$. Episodes of sepsis were similar in both groups averaging $20 \%$.

\section{Clinical Outcomes/End Points}

Further subgroup analysis of patients on cinacalcet who suffered the cardiac events (Table 5) indicated a significantly higher troponin I levels at baseline and 18 months, the latter highly significant $(\mathrm{p}<0.009)$. PTH levels on the other hand, were statistically more increased at baseline in these patients but this difference was not maintained at 18 months, likely demonstrating cinacalcet treatment effect.

\section{Discussion}

Our results demonstrated an association between reduction in PTH levels induced by cinacalcet and a decline in levels of Troponin I. This was observed in patients with severe secondary hyper parathyroidism after a relatively short period on treatment and with only modest improvement in their biochemical profiles. Moreover, the calcimimetic treatment was initiated late in the course of the disease and its dosing was not maximized (average daily dose $45 \mathrm{mg}$ ). Despite this, the findings suggest a possible link between cinacalcet-induced PTH reduction and a possible cardioprotective effect reflected by decreased troponin levels. There is supportive evidence in the literature for the role of PTH. Secondary hyper parathyroidism, increased the cardio vascular risk, seen in chronic kidney disease (CKD) with a number of plausible mechanisms [9]-[14]. These include direct effects on atherogenesis via vascular calcification and remodeling, induction of LVH, cardiac calcification and fibrosis, in addition to its key regulatory role on factors involved in mineral metabolism and homeostasis such as hypercalcemia, hyperphosphatemia, vitamin D deficiency, which may individually have direct detrimental myocardial ef- 
fects [11] [15]-[19]. Interestingly, PTH has also been implicated with predicting cardiovascular (CV) mortality even in normal individuals with plasma levels well within normal range [9].

Additionally, indirect evidence for the contributions of secondary hyperparathyroidism to cardiac disease comes from the noted association between serum alkaline phosphatase levels and coronary artery calcification in HD patients [11]. Moreover, measures which lower PTH levels, such as parathyroidectomy, have been associated with reduced vascular event rates and mortality in dialysis patients [2]. Similar findings have been reported with renal transplantation [20]. In addition, a lowered incidence of CV disease has also been observed with calcimimetics use, not only in patients with secondary hyperparathyroidism but also in those with primary hyperparathyroidism [18] [21]. The effects of calcimimetics remain controversial however, with some studies like the ADVANCE [22] showing promising results with reduced vascular calcification scores in HD patients, whereas others demonstrating no outcomes benefit as will be discussed. In the recently published metaanaysis by

Table 1. Basic characteristics of the cohort.

\begin{tabular}{ccc}
\hline & Cinacalcet N =63 & Control N $=40$ \\
\hline Age: mean (SD) (yrs) & $55.4(16.5)$ & $60.4(15)^{*}$ \\
Female: $\mathrm{n}(\%)$ & $33(52.4)$ & $17(42.5)^{*}$ \\
Duration of dialysis: mean (SD) months & $74(58)$ & $46(89)^{\dagger}$ \\
Hypertension: $\mathrm{n}(\%)$ & $49(77.8)$ & $35(80)^{*}$ \\
Diabetes: $\mathrm{n}(\%)$ & $28(44.4)$ & $21(52.5)^{*}$ \\
Ischemic heart diseases: $\mathrm{n}(\%)$ & $21(33.3)$ & $14(35)^{*}$ \\
\hline
\end{tabular}

${ }^{*} \mathrm{P}$ values NS ${ }^{\dagger} \mathrm{p}$ value $<0.05$

Table 2. Variation of parameters over the study duration.

\begin{tabular}{cccc}
\hline & baseline & 12 Month & 18 month \\
\hline Mean PTH (pmol/l) & $95.45 \pm 62$ & $88.06 \pm 68^{*}$ & $71.91 \pm 48^{\dagger}$ \\
Troponin I (ng/ml) & $0.05 \pm 0.11$ & $0.036 \pm 0.2^{*}$ & $0.025 \pm 0.03^{\dagger}$ \\
CRP (mg/l) & $16.24 \pm 19$ & $12.55 \pm 11^{*}$ & $7.7 \pm 9^{*}$ \\
Ca (mg/L) x Ph (mg/L) Mean (SD) & $3.89(1.28)$ & $2.87(2.03)^{\dagger+}$ & $1.91(1.97)^{\dagger+}$ \\
Cinacalcet dose (mg) & 0 & $37.9 \pm 19$ & $40.26 \pm 23$ \\
\hline
\end{tabular}

$\mathrm{p}$ value vs baseline ${ }^{*} \mathrm{NS} ;{ }^{\dagger} \mathrm{p}$ value $\leq 0.05 ;{ }^{\dagger \dagger} \mathrm{P}$ value $<0.001$.

Table 3. Changes of studied parameters over time after pairing patients as their own control.

\begin{tabular}{ccc}
\hline Baseline & 12 months & 18 months \\
\hline PTH (pmol/l) Mean (SD) 105.2 (70.1) & $95.7(72.7)(\mathbf{p m o l} / \mathbf{L})^{*}$ & $76.9(50.7)^{\dagger}(\mathbf{p m o l} / \mathbf{L})$ \\
Troponin I (ng/ml) Median (P25-P75) 0.02 (0.01 - 0.04) & $0.01(0.01-0.03)^{\dagger}(\mathbf{n g} / \mathbf{m l})$ & $0.02(0.01-0.02)^{\dagger}(\mathbf{n g} / \mathbf{m l})$ \\
CRP (mg/l) Median (P25-P75) 10.5 (5 - 20) & $7(3.75-17.25)^{*}(\mathbf{m g} / \mathbf{l})$ & $4(2-12.5)^{*}(\mathbf{m g} / \mathbf{l})$ \\
Cinacalcet dose (mg) & & $81.6 \%$ \\
30 mg 86.9\% & $79.6 \%$ & $18.4 \%$
\end{tabular}

p value vs baseline. ${ }^{*} \mathrm{NS} ;{ }^{\dagger} \mathrm{p}$ value $<0.05$.

Table 4. Rate of complications in cinacalcet versus control group.

\begin{tabular}{ccc}
\hline & Cinacalcet $\mathrm{N}=63 \mathrm{n}(\%)$ & Control N $=40 \mathrm{n}(\%)$ \\
\hline Overall mortality & $6(9.5)$ & $4(10)^{*}$ \\
Cardiac related mortality & $3(4.8)$ & $2(5)^{*}$ \\
Cardiac events & $21(33.3)$ & $27(67.5)^{\dagger}$ \\
CVA & $1(1.6)$ & $8(20)^{\dagger}$ \\
PVD & $4(6.3)$ & $7(17.5)^{ \pm}$ \\
Sepsis & $12(19)$ & $8(20)^{*}$ \\
\hline
\end{tabular}

${ }^{*} \mathrm{P}$ value NS, ${ }^{\dagger}$ pvalue $=0.001{ }^{ \pm} \mathrm{p}=0.07$ 
Table 5. Subgroup analysis of subjects with cardiovascular events.

\begin{tabular}{ccc}
\hline & CV Events N =48 & No CV Events N $=55$ \\
\hline Troponin at baseline $\mathrm{ng} / \mathrm{ml}$ & $0.05 \pm 0.11$ & $0.01 \pm 0.026^{\dagger}$ \\
Troponin at 18 months $\mathrm{ng} / \mathrm{ml}$ & $0.04 \pm 0.03$ & $0.023 \pm 0.03^{\dagger}$ \\
PTH at baseline $\mathrm{pmol} / \mathrm{L}$ & $95.45 \pm 62$ & $49.76 \pm 44^{\dagger}$ \\
PTH at 18 months $\mathrm{pmol} / \mathrm{L}$ & $71.91 \pm 48^{*}$ & $51.37 \pm 78^{*}$ \\
\hline
\end{tabular}

${ }^{*} \mathrm{P}$ value NS, ${ }^{\dagger} \mathrm{p}$ value $\leq 0.05$.

Zhang, 15 large studies in end-stage renal disease patients with secondary hyperparathyroidism were reviewed [12]. This trial demonstrated effective improvement of biochemical parameters by cinacalcet without increasing all-cause of mortality or adverse events but conversely no advantages were noted either. However, a major limitation of this trial was the few number of studies available as well as the small number of subjects and short duration of follow-up.

Similar findings were reported by Palmer [23] in another large meta-analysis with data from 18 trials, whereby the use of cinacalcet conferred no significant benefit for all-cause mortality in patients with stage 5D Chronic Kidney Disease. Nevertheless, the investigators reported “uncertain” effects on cardio vascular outcomes. The results are hence inconclusive since in addition as with all metaanalysis, their generalizability is limited by protocol herterogeneity and differences in study population. We must therefore seek evidence from randomized clinical trials, though there has been few.

Nonetheless, the long awaited EVOLVE trial [24] showed these same findings with similar mortality outcomes in patients on cinaclacet compared to those on conventional treatment. There were however, some limitations to this well designed and executed trial. These included a high dropout rate of $20 \%$ and an additional $20 \%$ of patients in placebo group who were actually receiving commercially available cinacalcet [25]. As importantly, the difference in age though small, may have had great importance in dialysis patients [25]. Hence post hoc secondary analysis might actually be signaling some benefits [25]. Namely after adjusting for age and other comorbidities, a significant reduction of $12 \%$ was observed in the composite end point of death or major CV events [25]. Furthermore, censoring of data at 6 months after drug discontinuation found 15\% reduction in primary composite and $17 \%$ in mortality with cinacalcet [25]. It therefore remains speculative whether the EVOLVE trial may in fact be marginally positive in favor of cinacalcet.

In our study, we noted a similar lack of cinacalcet treatment impact on all -cause and cardiac mortality. Interestingly however, our data demonstrated significantly lower cardiovascular event rates in the cinacalcet-treated group versus controls. This was seen despite longer dialysis vintage in the former which was not in their favor. There are some demographic variables in our study which may be potential confounders of the witnessed results, namely the older age of the control cohort and the higher proportion with diabetes. None of these differences however, achieved statistical significance. On the other hand, the cinacalcet group had more severe SHPT reflected by higher PTH levels which along with their longer duration on dialysis, may actually predispose to increased CV risk.

Moreover, it appears that the subgroup of patients with cardiac events had higher baseline troponin levels vs their non-cardiac events counterparts. Since troponin I is a specific biomarker for myocardial ischemia, this may explain in part their increased CV risk. It would hence be plausible that following troponin I trends may serve not only as possible marker of cinacalcet treatment effect on cardiac risk but suggests its utility as an overall prognostic indicator.

Finally, the paired group analysis showed an early drop in Troponin I levels at 12 months with non-significant changes in PTH levels, perhaps suggesting PTH-independent effects on the cardiac biomarker levels. The role of inflammation is possible, given the strong association described between chronic inflammatory markers (CRP) and disturbance of bone mineral metabolism in chronic hemodialysis patients [26]. This however, was not confirmed by our findings with no significant time variation in CRP levels. Unexplained in addition, is the nonsustained decline in troponin levels in this subgroup at 18 months, a time when PTH levels dropped significantly. These observations may be explained by additional unrelated factors or may have little clinical significance possibly due to our small sample size. All in all, given our study limitations which include the short duration, its retrospective nature, suboptimal cinacalcet dosing as well as late initiation, these findings require confirmation with appropriately designed prospective trials. 


\section{Conclusion}

Our results suggest an association between reduction in PTH levels induced by cinacalcet and a decline in levels of Troponin I with demonstrably lower cardiovascular event rates. This however was not linked to an observed mortality benefit in the treated group. There are some potential confounders which may account for our findings including possible selection bias, however, clearly the pathophysiologic mechanisms are plausible and observations are warranting further investigation. Moreover, the raised baseline troponins in the subgroup of cinacalcettreated patients with cardiac events may suggest utility of this biomarker in risk stratification. Large long-term placebo-controlled prospective trials are needed to explore this further and establish the clinical implications of calcimimetic treatment of secondary hyperparathyroidism on cardiovascular outcomes before the jury is out on cinacalcet.

\section{Acknowledgements}

We have no conflict of interest to disclose.

\section{References}

[1] Salgueira, M., del Toro, N., Moreno-Alba, R., Jimenez, E., Areste, N., et al. (2003) Vascular Calcification in the Uremic Patient: A Cardiovascular Risk? Kidney International, Supl, S119-S121.

[2] Costa-Hong, V., Jorgetti, V., Gowdak, L.H., Moyses, R.M., Krieger, E.M. and De Lima, J.J. (2007) Parathyroidectomy Reduces the Cardiovascular Events and Mortality in Renal Hyperparathyroidism. Surgery, 142, 699-703. http://dx.doi.org/10.1016/j.surg.2007.06.015

[3] Block, G.A., Zaun, D., Smits, G., Persky, M., Brillhart, S., et al. (2010) Cinacalcet Hydrochloride TNT Significantly Improves All Cause and Cardiovascular Survival in a Large Cohort of Hemodialysis Patients. Kidney International, 78, 578-599.

[4] Gaiki, M.R., Devita, M.V., Michelis, M.F., Panagopoulos, G. and Rosenstock, J.L. (2012) Troponin I as a Prognostic Marker of Cardiac Events in Symptomatic Hemodialysis Patients Using a Sensitive Trop I Assay. International Urology and Nephrology, 44, 1841-1845.

[5] Artunc, F., Mueller, C., Breidthardt, T., Twerenbold, R., Peter, A., Thamer, C., Weyrich, P., Haering, H.U. and Friedrich, B. (2012) Sensitive Troponins Which Suits Better for Hemodialysis Patients? Associated Factors and Prediction of Mortality. PLOS One, 7, Article ID: e47610.

[6] Flisinski, M., Strozecki, P., Stefanska, A., Zarzycka-Lindner, G., Brymora, A. and Manitius, J. (2007) Cardiac Troponin I in Patients with Chronic Kidney Disease Treated Conservatively or Undergoing Long-Term Haemodialysis. Kardiologia Polska, 65, 1068-1078.

[7] Kalaji, F.R. and Albitar, S. (2012) Predictive Value of Cardiac Troponin T and I in Hemodialysis Patients. Saudi Journal of Kidney Disease and Transplantation, 23, 939-945. http://dx.doi.org/10.4103/1319-2442.100868

[8] Geerse, D.A., Van Berkel, M., Vogels, S., Kooman, J.P., Konings, C.J. and Scharnhorst, V. (2012) Moderate Elevation of High Sensitivity Cardiac Troponin I and B Type Natriuretic Peptide in Chronic Hemodialysis Patients Are Associated with Mortality. Clinical Chemistry and Laboratory Medicine, 10, 1-8.

[9] Hagstrom, E., Hellman, P., Larsson, T.E., Ingelsso, E., Berglund, L., Sundstrom, J., Meihus, H., Held, C., Lind, L., Michaelsson, K. and Aenlov, J. (2009) Plasma Parathyroid Hormone and the Risk of Cardiovascular Mortality in the Community. Circulation, 119, 2765-2771. http://dx.doi.org/10.1161/CIRCULATIONAHA.108.808733

[10] Weiner, D.E., Tabatabai, S., Tighiouart, H., Elsayed, E., Bansal, N., et al. (2006) Cardiovascular Outcomes and AllCause Mortality: Exploring the Interaction between CKD and Cardiovascular Disease. American Journal of Kidney Diseases, 48, 392-401. http://dx.doi.org/10.1053/j.ajkd.2006.05.021

[11] Shantouf, R., Kovesdy, C.P., Kim, Y., Ahmadi, N., Luna, A., Luna, C., Rambod, M., Nissenson, A.R., Budoff, M.J. and Kalantar-Zadeh, K. (2009) Association of Serum Alkaline Phosphatise with Coronary Artery Calcification in Maintenance Hemodialysis Patients. Clinical Journal of the American Society of Nephrology, 6, 1106-1114. http://dx.doi.org/10.2215/CJN.06091108

[12] Zhang, Q., Li, M., You, L., Li, H.M., Ni, L., Gu, Y., Hao, C.M. and Chen, J. (2012) Effects and Safety of Calcimimetics in End Stage Renal Disease Patients with Secondary Hyperparathyroidism: A Meta-Analysis. PLoS One, 7, Article ID: e48070. http://dx.doi.org/10.1371/journal.pone.0048070

[13] Block, G.A., Martin, K.J., de Francisco, A.L., Turner, S.A., Avram, M.M., et al. (2004) Cinacalcet for Secondary Hyperparathyroidism in Patients Receiving Hemodialysis. The New England Journal of Medicine, 350, 1516-1525.

[14] Fukagawa, M., Yumita, S., Akizawa, T., Uchida, E., Tsukamoto, Y., et al. (2008) Cinacalcet (KRN1493) Effectively 
Decreases the Serum Intact PTH Level with Favourable Control of the Serum Phosphorous and Calcium Levels in Japanese Dialysis Patients. Nephrology Dialysis Transplantation, 23, 328-335.

[15] Saleh, F.N., Schirmer, H., Sundsfjord, J. and Jorde, R. (2003) Parathyroid Hormone and Left Ventricular Hypertrophy. European Heart Journal, 24, 2054-2060. http://dx.doi.org/10.1016/j.ehj.2003.09.010

[16] Johnson, D.W., Craven, A.M. and Isbel, N.M. (2007) Modification of Cardiovascular Risk in Hemodialysis Patients: An Evidence-Based Review. Hemodialysis International, 11, 1-14.

[17] Goodman, W.G., Goldin, J., Kuizon, B.D., Yoon, C., Gales, B., et al. (2000) Coronary Artery Calcification in Young Adults with End Stage Renal Disease Who Are Undergoing Dialysis. The New England Journal of Medicine, 342, 1478-1483.

[18] Andersson, P., Rydberg, E. and Willenheimer, R. (2004) Primary Hyperparathyroidism and Heart Disease: A Review. European Heart Journal, 25, 1776-1787. http://dx.doi.org/10.1016/j.ehj.2004.07.010

[19] Giovannucci, E., Liu, Y., Hollis, B.W. and Rimm, E.B. (2008) 25-Hydroxyvitamin D and Risk of Myocardial Infarction in Men: A Prospective Study. Archives of Internal Medicine, 168, 1174-1180. http://dx.doi.org/10.1001/archinte.168.11.1174

[20] Wolfe, R.H., Ashby, V.B., Milford, E.L., Ojo, A.O., Ettenger, R.E., Agodoa, L.Y., Held, P.J. and Port, F.K. (1999) Comparison of Mortality in all Patients on Dialysis, Patients on Dialysis Awaiting Transplantation and Recipients of a First Cadaveric Transplant. The New England Journal of Medicine, 341, 1725-1730. http://dx.doi.org/10.1056/NEJM199912023412303

[21] Cunninghm, J., Danese, M., Olson, K., Klassen, P. and Chertow, G.M. (2005) Effects of the Calcimimetic Cinacalcet $\mathrm{HCl}$ on Cardiovascular Disease, Fracture, and Health Related Quality of Life in Secondary Hyperparathyroidism. Kidney International, 68, 1793-1800. http://dx.doi.org/10.1111/j.1523-1755.2005.00596.X

[22] Raggi, P., Chertow, G.M., Torres, P.U., Csiky, B., Naso, A., et al. (2011) The ADVANCE Study: A Randomised Study to Evaluate the Effects of Cinacalcet Plus Low Dose Vitamin D on Vascular Calcification in Patients on Hemodialysis. Nephrology Dialysis Transplantation, 26, 1327-1339.

[23] Palmer, S.C., Nistor, I., Craig, J.C., Pellegrini, F., Messa, P., Tonelli, M., Covic, A. and Strippoli, G.F.M. (2013) Cinacalcet in Patients with Chronic Kidney Disease: A Cumulative Meta-Analysis of Randomised Controlled Trials. PLOS Medicine, 10, Article ID: e1001436. http://dx.doi.org/10.1371/journal.pmed.1001436

[24] Glenn, M.C., Geoffrey, A.B., Ricardo, C.R., Tilman, B.D., Jurgen, F., willim, G.G., Charles, A.H., Yumi, K., Gerard, M.L., Kenneth, W.M., Christian, T.H., Sharon, M.M., Marie, L.T., David, C.W. and Patrick, S.P. (2012) Evaluation of Cinacalcet Hydrochloride Therapy to Lower Cardio Vascular Events. The New England Journal of Medicine, 2, 898905.

[25] The Evolve Trial Investigators (2012) Effects of Cinacalcet on Cardiovascular Disease in Patients Undergoing Dialysis. The New England Journal of Medicine, 367, 2482-2494. http://dx.doi.org/10.1056/NEJMoa1205624

[26] Lee, C.T., Tsai, Y.C., Ng, H.Y., Su, Y., Lee, W.C., Lee, L.C., Chiou, T.T., Liao, S.C. and Hsu, K.T. (2009) Association between C-Reactive Protein and Biomarkers of Bone and Mineral Metabolism in Chronic Hemodialysis Patients: A Cross-Sectional Study. Journal of Renal Nutrition, 19, 220-227. 